\title{
Markov-Bernstein Type Inequalities for Classes of Polynomials with Restricted Zeros
}

\author{
Peter Borwein \\ Department of Mathematics \\ Dalhousie University \\ Halifax, Nova Scotia \\ Canada B3H 3J5 \\ Tamás Erdélyi \\ Department of Mathematics \\ The Ohio State University \\ 231 West Eighteenth Avenue \\ Columbus, Ohio 43210 \\ USA
}


Abstract. We prove that there exists an absolute constant $c>0$ such that

$$
\left|p^{\prime}(y)\right| \leq c \min \left\{n(k+1),\left(\frac{n(k+1)}{1-y^{2}}\right)^{1 / 2}\right\} \max _{-1 \leq x \leq 1}|p(x)|, \quad-1 \leq y \leq 1
$$

for every real algebraic polynomial of degree at most $n$ having at most $k$ zeros in the open unit disk $\{z \in \mathbb{C}:|z|<1\}$. This inequality, which has been conjectured for at least a decade, improves and generalizes several earlier results. Up to the multiplicative absolute constant $c$, it is a sharp generalization of both Markov's and Bernstein's inequalities.

\section{Introduction, Notations}

Bernstein's inequality [LO1, pp. 39-41] asserts that

$$
\max _{-\pi \leq t \leq \pi}\left|p^{\prime}(t)\right| \leq n \max _{-\pi \leq t \leq \pi}|p(t)|
$$

for every $p \in \mathcal{T}_{n}$, where $\mathcal{T}_{n}$ denotes the set of all trigonometric polynomials of degree at most $n$ with real coefficients. The corresponding algebraic result [LO1, pp. 39-41], known as Markov's inequality, states that

$$
\max _{-1 \leq x \leq 1}\left|p^{\prime}(x)\right| \leq n^{2} \max _{-1 \leq x \leq 1}|p(x)|
$$

for all $p \in \mathcal{P}_{n}$, where $\mathcal{P}_{n}$ denotes the set of all algebraic polynomials of degree at most $n$ with real coefficients. The Chebyshev polynomials $Q_{n} \in \mathcal{T}_{n}$ and $T_{n} \in \mathcal{P}_{n}$ defined by

$$
Q_{n}(t):=\cos (n t+\alpha), \quad \alpha \in \mathbb{R}
$$

and

$$
T_{n}(x):=\cos (n \arccos x), \quad-1 \leq x \leq 1
$$

show that inequalities (1.1) and (1.2) are sharp. The substitution $x=\cos t$ in (1.1), together with (1.2), yields

$$
\left|p^{\prime}(y)\right| \leq \min \left\{n^{2}, \frac{n}{\sqrt{1} x^{2}}\right\} \max _{1<x<1}|p(x)|, \quad-1 \leq y \leq 1
$$


for every $p \in \mathcal{P}_{n}$. Markov-Bernstein type inequalities in weighted spaces and in $L_{p}$ norms play a key role in proving inverse theorems of approximation and of course have their own intrinsic interest.

Denote by $\mathcal{P}(n, k)$ the set of all $p \in \mathcal{P}_{n}$ having at most $k$ zeros (by counting multiplicities) in the open unit disk $\{z \in \mathbb{C}:|z|<1\}$. Markov-Bernstein type inequalities for constrained polynomials have been the subject of many research papers and the classes $\mathcal{P}(n, k), 0 \leq k \leq n$, have been of special interest. One might correctly suspect that the restrictions on the zeros of a polynomial imply an improvement in inequality (1.5). In 1940 Erdős [ERDŐS] proved that there is an absolute constant $c>0$ such that

$$
\left|p^{\prime}(y)\right| \leq \min \left\{\frac{e n}{2}, \frac{c \sqrt{n}}{\left(1-y^{2}\right)^{2}}\right\} \max _{-1 \leq x \leq 1}|p(x)|, \quad-1 \leq y \leq 1
$$

for every $p \in \mathcal{P}(n, 0)$ having only real zeros. By taking the polynomials $p_{n} \in \mathcal{P}(n, 0)$ defined by $p_{n}(x):=(1+x)^{n-1}(1-x)$, it is easy to see that the constant $e / 2$ in (1.6) is asymptotically sharp. In 1963 G. G. Lorentz [LO2] showed that there is an absolute constant $c>0$ such that

$$
\left|p^{\prime}(y)\right| \leq c \min \left\{n, \frac{\sqrt{n}}{\sqrt{1-y^{2}}}\right\} \max _{-1 \leq x \leq 1}|p(x)|, \quad-1 \leq y \leq 1
$$

for every $p \in \mathcal{P}_{n}$ of the form

$$
p(x)=\sum_{j=0}^{n} a_{j}(1+x)^{j}(1-x)^{n-j} \text { with all } a_{j} \geq 0 \text { or all } a_{j} \leq 0 .
$$

By an observation of G. G. Lorentz [SCH], every $p \in \mathcal{P}(n, 0)$ is of the form (1.8), therefore (1.7) holds for every $p \in \mathcal{P}(n, 0)$. Inequality (1.7) is sharp up to the multiplicative absolute constant $c>0$; namely it was shown in [ER1] that there is an absolute constant $c>0$ such that

$$
\sup _{p \in \mathcal{P}(n, 0)} \frac{\left|p^{\prime}(y)\right|}{\max _{-1 \leq x \leq 1}|p(x)|} \geq c \min \left\{n, \frac{\sqrt{n}}{\sqrt{1-y^{2}}}\right\}
$$

for every $n \in \mathbb{N}$ and $y \in[-1,1]$. In 1972 Scheick $[\mathrm{SCH}]$ found the best possible constant in Lorentz's Markov-type inequality. Extending Erdős's Markov-type inequality, he proved that

$$
\max \left|p^{\prime}(x)\right|<\frac{e n}{\max }|p(x)|
$$


for every $p \in \mathcal{P}_{n}$ of the form (1.8), hence for every $p \in \mathcal{P}(n, 0)$. In 1980 Szabados and Varma showed that there is a constant $c(k)>0$ depending only on $k$ such that

$$
\max _{-1 \leq x \leq 1}\left|p^{\prime}(x)\right| \leq c(k) n \max _{-1 \leq x \leq 1}|p(x)|
$$

for every $p \in \mathcal{P}(n, k), 0 \leq k \leq n$, having only real zeros. Subsequently Máté proved that

$$
\max _{-1 \leq x \leq 1}\left|p^{\prime}(x)\right| \leq 6 n \exp (\pi \sqrt{k}) \max _{-1 \leq x \leq 1}|p(x)|
$$

for every $p \in \mathcal{P}(n, k), 1 \leq k \leq n$, having $n-k$ zeros in $\mathbb{R} \backslash(-1,1)$. Szabados' conjecture, proved by P. Borwein, [BO] establishes the Markov-type inequality

$$
\max _{-1 \leq x \leq 1}\left|p^{\prime}(x)\right| \leq 9 n(k+1) \max _{-1 \leq x \leq 1}|p(x)|
$$

for every $p \in \mathcal{P}(n, k), 0 \leq k \leq n$, having $n-k$ zeros in $\mathbb{R} \backslash(-1,1)$. Inequality (1.13) was extended in [ER2] to all $p \in \mathcal{P}(n, k), 0 \leq k \leq n$. Another proof of (1.13) for all $p \in \mathcal{P}(n, k), 0 \leq k \leq n$, is obtained in [ER3] with the constant 11 instead of 9 . The fact that (1.13) is sharp up to the multiplicative constant was shown by Szabados [SZA, Example 1]. While (1.13) is essentially sharp, it is only a good estimate for $\left|p^{\prime}(y)\right|$ with $|y|$ close to 1 .

It was proved in [ER-SZA] that there is an absolute constant $c>0$ such that

$$
\left|p^{\prime}(y)\right| \leq \frac{c \sqrt{n}(k+1)^{2}}{\sqrt{1-y^{2}}} \max _{-1 \leq x \leq 1}|p(x)|, \quad-1<y<1
$$

for every $p \in \mathcal{P}(n, k), 0 \leq k \leq n$. Subsequently it was shown in [ER3] that there is an absolute constant $c>0$ such that

$$
\left|p^{\prime}(y)\right| \leq \frac{c \sqrt{n(k+1)}}{1-y^{2}} \max _{-1 \leq x \leq 1}|p(x)|, \quad-1<y<1
$$

for every $p \in \mathcal{P}(n, k), 0 \leq k \leq n$. When $y=0$, inequality (1.15) is sharp up to the multiplicative constant $c>0$; namely it was verified in [ER2] that there is an absolute constant $c>0$ such that

$$
\sup _{p \in \mathcal{P}(n, k)} \frac{\left|p^{\prime}(0)\right|}{\max _{-1 \leq x \leq 1}|p(x)|} \geq c \sqrt{n(k+1)}
$$

for every $0 \leq k \leq n$. The unpleasant thing about the Berstein-type inequalities (1.14) and (1.15) is the fact that none of them matches inequality (1.5) in the unrestricted case $k=n$ (note that $\mathcal{P}(n, n)=\mathcal{P}_{n}$ ). To formulate a Markov-Bersnstein type inequality for $\mathcal{P}(n, k), 0 \leq k \leq n$, which contains all of the earlier results as 
Theorem. There is an absolute constant $c>0$ such that

$$
\left|p^{\prime}(y)\right| \leq c \min \left\{n(k+1),\left(\frac{n(k+1)}{1-y^{2}}\right)^{1 / 2}\right\} \max _{-1 \leq x \leq 1}|p(x)|, \quad-1 \leq y \leq 1
$$

for every $p \in \mathcal{P}(n, k), 0 \leq k \leq n$.

The purpose of this paper is to prove the above theorem, which seems to be the "right" Markov-Bernstein type inequality for the classes $\mathcal{P}(n, k), 0 \leq k \leq n$. The proof relies on a series of lemmas, some of which are interesting for their own merit.

\section{Proof of the Theorem}

First we prove the following Bernstein-type inequality for trigonometric polynomials $p \in \mathcal{T}_{n}$ having $2 n-2 k$ zeros at 0 .

Theorem 1. Let $0 \leq k \leq n, n \geq 1$ be integers. There is an absolute constant $c_{1}>0$ such that

$$
\max _{t \in \mathbb{R}}\left|p^{\prime}(t)\right| \leq c_{1} \sqrt{n(k+1)} \max _{t \in \mathbb{R}}|p(t)|
$$

for every $p \in \mathcal{T}_{n}$ of the form

$$
p(t)=(\sin (t / 2))^{2 n-2 k} q(t), \quad q \in \mathcal{T}_{k} .
$$

Theorem 1 implies immediately the following result for higher derivatives by induction on $s$.

Corollary 2. Let $0 \leq k \leq n, n \geq 1$ and $s \geq 1$ be integers. Then

$$
\max _{t \in \mathbb{R}}\left|p^{(s)}(t)\right| \leq \prod_{j=1}^{s}\left(c_{1} \sqrt{n(k+j)}\right) \max _{t \in \mathbb{R}}|p(t)|
$$

for every $p \in \mathcal{T}_{n}$ of the form (2.2).

The proof of Theorem 1 rests on the following.

Theorem 3. Let $0 \leq k \leq n$ and $n \geq 1$ be integers. There is an absolute constant $c_{2}>0$ such that

$$
|p(z)| \leq c_{2} \max _{t \in \mathbb{R}}|p(t)|
$$

for every $p \in \mathcal{T}_{n}$ of the form (2.2), and for every $z \in \mathbb{C}$ such that $|\operatorname{Im}(z)| \leq$ $(n(k+1))^{-1 / 2}$. 
Lemma 4. Let $a<b$ and $r>0$ be arbitrary real numbers. Assume that $P \in \mathcal{P}_{2 n}$ and

$$
|P(b)|=\max _{a \leq x \leq b}|P(x)|
$$

Then $P$ has at most $c_{3} n r^{1 / 2}(b-a)^{-1 / 2}$ zeros (by counting multiplicities) in the interval $[b-r, b]$ with some $0<c_{3} \leq 4$.

Lemma 4 is proved in [ER4, Corollary 1] with a slightly larger constant. The fact that $c_{3} \leq 4$ was pointed out by Lorentz and von Golitschek. Our next lemma is a special case of [BO-ER, Theorem 3.2].

Lemma 5. Let $0 \leq k \leq n$ and $n \geq 1$ be integers. For every $c_{4}>0$ there is a constant $c_{5}=c_{5}\left(c_{4}\right)$ depending only on $c_{4}$ such that

$$
\max _{a \leq x \leq b+c_{4}(b-a)(n(k+1))^{-1}}|P(x)| \leq c_{5} \max _{a \leq x \leq b}|P(x)|
$$

holds for every $P \in \mathcal{P}_{2 n}$ of the form

$$
P(x)=(x-a)^{2 n-2 k} Q(x), \quad Q \in \mathcal{P}_{2 k} .
$$

¿From Lemmas 4 and 5 we deduce

Lemma 6. Let $0 \leq k \leq n, n \geq 1$ be integers. There is an absolute constant $c_{6}>0$ such that

$$
\max _{-1 \leq x \leq 1+(n(k+1))^{-1}}|P(x)| \leq c_{6} \max _{-1 \leq x \leq 1}|P(x)|
$$

for every polynomial of the form

$$
P(x)=(x-a)^{2 n-2 k} Q(x), \quad Q \in \mathcal{P}_{2 k}, \quad a \in[-1,1]
$$

Proof. If $n / 2 \leq k \leq n$, then inequality (2.8) holds for every $P \in \mathcal{P}_{2 n}$ by Bernstein's inequality [LO1, pp. 42-43]. Therefore, in the sequel we assume that $0 \leq k \leq n / 2$. Let $b \geq 1$ be the smallest real number for which 
Since $0 \leq k \leq n / 2, P$ has $2 n-2 k \geq n$ zeros at $a$. On the other hand, Lemma 4 implies that $P$ has at most $c_{3} n(b-a)^{1 / 2}(b+1)^{-1 / 2}$ zeros (by counting multiplicities) in $[a, b]$, hence

$$
c_{3} n(b-a)^{1 / 2}(b+1)^{-1 / 2} \geq n
$$

thus

$$
(b-a)^{-1} \leq \frac{c_{3}^{2}}{2}=: c_{7}
$$

Now Lemma 5 and inequality (2.11) yield that

$$
\begin{aligned}
& \max _{-1 \leq x \leq 1+(n(k+1))^{-1}}|P(x)| \leq \max _{a \leq x \leq b+(n(k+1))^{-1}}|P(x)| \\
& \leq \max _{a \leq x \leq b+c_{7}(b-a)(n(k+1))^{-1}} P(x) \leq c_{6} \max _{a \leq x \leq b}|P(x)|=c_{6} \max _{-1 \leq x \leq 1}|P(x)|,
\end{aligned}
$$

where $c_{6}=c_{5}\left(c_{7}\right)>0$ is an absolute constant, and the lemma is proved.

¿From Lemma 6 we easily obtain.

Lemma 7. Let $0 \leq k \leq n, n \geq 1$ be integers. There is an absolute constant $c_{8}>0$ such that

$$
|p(i \delta)| \leq c_{8} \max _{t \in \mathbb{R}}|p(t)|
$$

( $i$ denotes the imaginary unit) for every $p \in \mathcal{T}_{n}$ of the form

$$
p(t)=(\sin ((t-\alpha) / 2))^{2 n-2 k} q(t), \quad q \in \mathcal{T}_{k}, \quad \alpha \in \mathbb{R}
$$

and for every $\delta \in \mathbb{R}$ such that

$$
|\delta| \leq(n(k+1))^{-1 / 2}
$$

Proof. Let

$$
\tilde{p}(t):=p(t) p(-t)=\frac{1}{4^{n-k}}(\cos t-\cos \alpha)^{2 n-2 k} q(t) q(-t) .
$$

Since $q(t) q(-t) \in \mathcal{T}_{2 k}$ is an even trigonometric polynomial, by the subsitutions $x=\cos t$ and $a=\cos \alpha$, we obtain that 
For the sake of brevity let

$$
P(x):=(x-a)^{2 n-2 k} Q(x) .
$$

Now (2.15) - (2.18) and Lemma 6 yield

$$
\begin{aligned}
& |p(i \delta)|^{2}=|p(i \delta) p(-i \delta)|=|\tilde{p}(i \delta)| \\
& =\left|(\cosh \delta-a)^{2 n-2 k} Q(\cosh \delta)\right| \leq \max _{-1 \leq x \leq 1+\delta^{2}}|P(x)| \\
& \leq c_{6} \max _{-1 \leq x \leq 1}|P(x)|=c_{6} \max _{t \in \mathbb{R}}|\tilde{p}(t)| \\
& =c_{6} \max _{t \in \mathbb{R}}|p(t) p(-t)| \leq c_{6} \max _{t \in \mathbb{R}}|p(t)|^{2},
\end{aligned}
$$

and the lemma is proved.

Theorem 3 follows from Lemma 7 immediately, while Theorem 1 can be obtained from Theorem 3 by the Cauchy integral formula. Corollary 2, which follows from Theorem 1 by induction on $s$, plays a key role in the proof of our next lemma.

Lemma 8. Let $0 \leq k \leq n, n \geq 1$ be integers, let $p \in \mathcal{T}_{n}$ be of the form

$$
p(t)=(\sin (t / 2))^{2 n-2 k} q(t), \quad q \in \mathcal{T}_{k},
$$

let $t_{0} \in \mathbb{R}$ be such that

$$
p\left(t_{0}\right)=\max _{t \in \mathbb{R}}|p(t)|=1
$$

Then $p$ has at most $2 e c_{1} \sqrt{n(k+1)} h$ zeros (by counting multiplicities) in the interval

$\left[t_{0}-h, t_{0}+h\right]$ for any $0<h<\left(2 e c_{1}\right)^{-1}(k+1)^{1 / 2} n^{-1 / 2}$, where $c_{1}>0$ is the same as in Theorem 1 (and Corollary 2).

Proof. For the sake of brevity let

$$
s:=\left[2 e c_{1} \sqrt{n(k+1)} h\right]+1,
$$

where $c_{1}>0$ is the same as in Theorem 1. Assume that we can find $t_{1}<t_{2}<$ $\cdots<t_{\nu}$ in $\left[t_{0}-h, t_{0}+h\right]$ such that $p$ has $\mu_{i}$ repeated zeros at $t_{i}, 1 \leq i \leq \nu$, and $\sum_{i=1}^{\nu} \mu_{i}=s$. Now let

$$
\Omega(x):=\prod^{\nu}\left(x-t_{i}\right)^{\mu_{i}} .
$$


Then, by a well-known relation for the remainder of the Hermite interpolation polynomial, there exists a $\xi \in\left[t_{1}, t_{\nu}\right] \subset\left[t_{0}-h, t_{0}+h\right]$ such that

$$
p\left(t_{0}\right)-H\left(t_{0}\right)=\frac{1}{s !} p^{(s)}(\xi) \Omega\left(t_{0}\right)
$$

where $H \in \mathcal{P}_{s-1}$ and $H^{(j)}\left(t_{i}\right)=p^{(j)}\left(t_{i}\right)=0$ for every $1 \leq i \leq \nu$ and $0 \leq j \leq \mu_{i}-1$, so $H \equiv 0$. Hence, by $s !>(s / e)^{s}$ and from $(2.21)-(2.24)$, we can deduce that

$$
\begin{aligned}
& \left|p^{(s)}(\xi)\right| \geq s ! h^{-s}>\left(\frac{s}{e}\right)^{s}\left(\frac{2 e c_{1} \sqrt{n(k+1)}}{s}\right)^{s} \\
& \geq\left(2 c_{1}\right)^{s}(n(k+1))^{s / 2} \geq \prod_{j=1}^{s}\left(c_{1} \sqrt{n(k+j)}\right) \\
& =\prod_{j=1}^{s}\left(c_{1} \sqrt{n(k+j)}\right) \max _{t \in \mathbb{R}}|p(t)|
\end{aligned}
$$

for any $0<h<\left(2 e c_{1}\right)^{-1}(k+1)^{1 / 2} n^{-1 / 2}$ (these bounds for $h$ imply $1 \leq s \leq k+1$ by (2.22), which is used in the last inequality). Since (2.25) constradicts Corollary 2, our assumption is false, and the lemma is proved.

By the substitution $x+1=2 \sin ^{2}(t / 2)$ (i.e. $x=-\cos t$ ), from Lemma 8 we obtain

Corollary 9. Let $0 \leq k \leq n$ and $n \geq 1$ be integers, let $p \in \mathcal{P}_{n}$ be of the form

$$
p(x)=(x+1)^{n-k} q(x), \quad q \in \mathcal{P}_{k},
$$

and let

$$
|p(1)|=\max _{-1 \leq x \leq 1}|p(x)|
$$

Then $p$ has at most $4 e c_{1} \sqrt{n(k+1) h}$ zeros in $[1-h, 1]$ for any

$$
0<h<\left(4 e c_{1}\right)^{-2}(k+1) n^{-1}
$$

Our next lemma constructs a polynomial with some nice properties, which will 
Lemma 10. Let $1 \leq k \leq m \leq n, m+k \leq n$ be integers, let $0<c_{9} \leq 1$ be real and let

$$
y:=1-\frac{c_{9} k}{4 n} \quad \text { and } \quad 0<z \leq 1-\frac{c_{9} m}{n} .
$$

Let $Q_{2 k}$ be the Chebyshev polynomial $T_{2 k}=\cos (2 k \arccos x)$ transformed linearly from $[-1,1]$ to $[y, 1]$. Denote the zeros of $Q_{2 k}$ by

$$
(1>) x_{1}>x_{2}>\cdots>x_{k}>\tilde{x}_{k}>\tilde{x}_{k-1}>\ldots \tilde{x}_{1}(>y)
$$

and define

$$
P(x):=(x+1)^{n-m-k}(x-z)^{m} \prod_{j=1}^{k}\left(x-x_{j}\right) .
$$

Let $x_{0}:=1$ and $x_{k+1}:=y$. Let $\xi_{j} \in\left(x_{j}, x_{j-1}\right], j=1,2, \ldots, k+1$, be the (only) point for which

$$
\left|P\left(\xi_{j}\right)\right|=\max _{x_{j} \leq x \leq x_{j-1}}|P(x)| .
$$

Then

$$
\left|P\left(\xi_{j}\right)\right|<\left|P\left(\xi_{j+1}\right)\right|, \quad j=1,2, \ldots, k .
$$

Proof. We have

$$
\begin{aligned}
& P(x)=(x+1)^{n-m-k}(x-z)^{m} \prod_{j=1}^{k}\left(x-x_{j}\right) \\
& =(x+1)^{n-m-k}(x-z)^{m} \prod_{j=1}^{k}\left(x-\tilde{x}_{j}\right)^{-1} Q_{2 k}(x) \\
& =(x+1)^{n-m-k}(x-z)^{m}(x-y)^{-k} \prod_{j=1}^{k} \frac{x-y}{x-\tilde{x}_{j}} Q_{2 k}(x) .
\end{aligned}
$$

For the sake of brevity let

$$
F(x):=\prod_{j=1}^{k} \frac{x-y}{x-\tilde{x}_{j}}
$$

and 
Then $P=F G Q_{2 k}$. Since $Q_{2 k}$ equioscillates $k+1$ times on $[(1+y) / 2,1]$, it is sufficient to prove that both $F$ and $G$ are decreasing on $[(1+y) / 2,1]$. Since each of its factors is decreasing on $[(1+y) / 2, \infty)$, so is $F$. To show that $G$ is decreasing on $[(1+y) / 2,1]$ we write

$$
G=G_{1} G_{2}
$$

where

$$
G_{1}(x):=(x+1)^{n-m-k}(x-y)^{-k / 2}
$$

and

$$
G_{2}(x):=(x-z)^{m}(x-y)^{-k / 2} .
$$

We have

$$
\begin{aligned}
G_{1}^{\prime}(x) & =\frac{(x+1)^{n-m-k-1}(x-y)^{k / 2-1}\left((n-m-k)(x-y)-\frac{k}{2}(x+1)\right)}{(x-y)^{k}} \\
& <(x+1)^{n-m-k-1}(x-y)^{-k / 2-1}\left(n \frac{c_{9} k}{2 n}-\frac{k}{2}\right) \leq 0
\end{aligned}
$$

and

$$
\begin{aligned}
G_{2}^{\prime}(x) & =\frac{(x-z)^{m-1}(x-y)^{k / 2-1}\left(m(x-y)-\frac{k}{2}(x-z)\right)}{(x-y)^{k}} \\
& <(x-z)^{m-1}(x-y)^{-k / 2-1}\left(m \frac{c_{9} k}{4 n}-\frac{k}{2} \frac{c_{9} m}{2 n}\right)=0
\end{aligned}
$$

for every $x \in[(1+y) / 2,1]$, and the lemma is proved.

Lemma 11. Let $k, m, n, c_{9}, y, z, x_{j}(j=1,2, \ldots, k)$ and $P$ be the same as in Lemma 10. Let

$$
P^{*}(x)=(x+1)^{n-m-k}(x-z)^{m} Q^{*}(x), \quad Q^{*} \in \mathcal{P}_{k}
$$

be the constrained Chebyshev polynomials of degree $n$ on $[y, 1]$, which equioscillates $k+1$ times on $[y, 1]^{1}$. Denote the zeros of $P^{*}$ on $(y, 1)$ by $1>y_{1}>y_{2}>\cdots>$ $y_{k}(>y)$. Then $y_{j} \leq x_{j}$ for every $j=1,2, \ldots, k$.

\footnotetext{
${ }^{1}$ It is well-known from the theory of Chebyshev approximation that there is a "constrained Chebyshev polynomial" $P^{*} \in \mathcal{P}_{n}$ of the form (2.40) such that $P^{*}\left(\gamma_{j}\right)=(-1)^{k+1-j} \max _{y \leq x \leq 1}\left|P^{*}(x)\right|$
} 
Proof. This follows from Lemma 10, by proceeding exactly in the same way as in the proof of [BO, Lemma 4]. Suppose that the statement of the lemma is false. Choose the smallest $j$ for which $y_{j}>x_{j}$. Then pick $\eta$ so that

$$
\max _{x_{j} \leq x \leq 1}\left|\eta P^{*}(x)\right|=\max _{x_{j} \leq x \leq 1}|P(x)|
$$

(We will specify the sign of $\eta$ later.) We can deduce from the equioscillation of $P^{*}$ that $\eta P^{*}-P$ has at least $j-1$ zeros on $[\beta, 1]$, where $\beta$ is the smallest number greater than $y_{j}$, where $\left|\eta P^{*}\right|$ achieves its maximum on $[y, 1]$. From Lemma 10 we deduce that $\eta P^{*}-P$ has at least $k-j$ zeros on $[y, \alpha)$, where $\alpha:=\xi_{j+1}($ see $(2.30))$. We need only to observe that if we choose the sign of $\eta$ so that

$$
\operatorname{sign}\left(\eta P^{*}(\beta)\right)=-\operatorname{sign}(P(\alpha))
$$

then $\eta P^{*}-P$ must have at least two zeros in $(\alpha, \beta)$. Thus, together with the $n-m-k$ zeros at -1 and $m$ zeros at $z, \eta P^{*}-P$ has at least

$$
(n-m-k)+m+(j-1)+(k-j)+2=n+1
$$

zeros, a contradiction. Therefore $y_{j} \leq x_{j}$ for every $j=1,2, \ldots, k$, and the lemma is proved.

¿From Lemma 11 we prove the following

Lemma 12. Let $k, m, n, c_{9}, y$ and $z$ be the same as in Lemma 10 and 11. There is a constant $c_{10}=c_{10}\left(c_{9}\right)>0$ depending only on $c_{9}$ such that

$$
|p(\alpha)| \leq c_{10} \max _{y \leq x \leq 1}|p(x)|
$$

for every $p$ of the form

$$
p(x)=(x+1)^{n-m-k}(x-z)^{m} q(x), \quad q \in \mathcal{P}_{k},
$$


Proof. By a compactness argument and a variational method it is routine to show that it is sufficient to prove $(2.43)$ for $p=P^{*}$, where $P^{*}$ is the constrained Chebyshev polynomial of degree $n$ on $[y, 1]$ defined in Lemma 11. Recalling the definition of $y$ and $z$, and using Lemma 10, (2.45) and the well-known formula

$$
T_{2 k}(x)=\frac{1}{2}\left(\left(x+\sqrt{x^{2}-1}\right)^{2 k}+\left(x-\sqrt{x^{2}-1}\right)^{2 k}\right), \quad x \in \mathbb{R} \backslash(-1,1)
$$

for the Chebyshev polynomial of degree $2 k$ on $[-1,1]$, we obtain

$$
\begin{aligned}
& \frac{\left|P^{*}(\alpha)\right|}{\max _{y \leq x \leq 1}\left|P^{*}(x)\right|}=\frac{\left|P^{*}(\alpha)\right|}{\left|P^{*}(1)\right|} \\
& =\left(\frac{\alpha+1}{2}\right)^{n-m-k}\left(\frac{\alpha-z}{1-z}\right)^{m} \prod_{j=1}^{k} \frac{\alpha-y_{j}}{1-y_{j}} \\
& \leq\left(\frac{\alpha+1}{2}\right)^{n-m-k}\left(\frac{\alpha-z}{1-z}\right)^{m} \prod_{j=1}^{k} \frac{\alpha-x_{j}}{1-x_{j}} \\
& \leq\left(\frac{\alpha+1}{2}\right)^{n-m-k}\left(\frac{\alpha-z}{1-z}\right)^{m} \frac{Q_{2 k}(\alpha)}{Q_{2 k}(1)} \\
& \leq\left(1+\frac{1}{2 n k}\right)^{n-m-k}\left(1+\frac{1}{c_{9} m k}\right)^{m} \frac{T_{2 k}\left(1+8 c_{9}^{-1} k^{-2}\right)}{T_{2 k}(1)} \leq c_{10},
\end{aligned}
$$

where $Q_{2 k}$ is the Chebyshev polynomial $T_{2 k}=\cos (2 k \arccos x)$ transformed linearly from $[-1,1]$ to $[y, 1]$, and $c_{10}$ depends only on $c_{9}$. The lemma is now proved.

Lemma 12 and Corollary 9 allow us to prove the following.

Lemma 13. Let $1 \leq k \leq n, 0 \leq m \leq n-k$ be integers, let $-1 \leq a \leq 1$. There is an absolute constant $c_{11}>0$ such that

$$
|p(\alpha)| \leq c_{11} \max _{-1 \leq x \leq 1}|p(x)|
$$

for every $p \in \mathcal{P}_{n}$ of the form

$$
p(x)=(x+1)^{n-m-k}(x-a)^{m} q(x), \quad q \in \mathcal{P}_{k}
$$

and for every

$$
\alpha \in\left[1,1+(n k)^{-1}\right] .
$$

Proof. Without loss of generality we may assume that

$$
|p(1)|=\max |p(x)| .
$$


Any other case can be reduced to this by a linear transformation. We distinguish two cases.

Case 1: $1 \leq k \leq m$. We show that there is an absolute constant $c_{9} \in(0,1]$ such that

$$
a<1-\frac{c_{9} m}{n}
$$

Indeed, let $0<c_{9}<\left(4 e c_{1}\right)^{-2}$. By Corollary $9 p$ has at most

$$
4 e c_{1} \sqrt{n(k+m+1) c_{9} m n^{-1}}<8 e c_{1} \sqrt{c_{9}} m
$$

zeros (by counting multiplicities) in $\left[1-c_{9} m n^{-1}, 1\right]$. Therefore $(2.51)$ holds with $c_{9}=\left(8 e c_{1}\right)^{-2}$, since $p$ has a zero at $a$ with multiplicity $m$. Since $(2.51)$ holds, Lemma 12 can be applied to yield the desired result.

Case 2: $0 \leq m<k$. Now $p \in \mathcal{P}_{n}$ has all but $m+k<2 k$ zeros at -1 , hence [BO-ER, Theorem 3.2] (cf. Lemma 5) gives the conclusion of the lemma.

Transforming the result of Lemma 13 linearly from $[-1,1]$ to $[-|b|, 1](-1 \leq b \leq$ 1), we immediately obtain

Corollary 14. Let $1 \leq k \leq n, 0 \leq m \leq n-k$ be integers and let $-1 \leq b \leq 1$. There is an absolute constant $c_{12}>0$ such that

$$
|p(\alpha)| \leq c_{12} \max _{-|b| \leq x \leq 1}|p(x)| \leq c_{12} \max _{-1 \leq x \leq 1}|p(x)|
$$

for every $p \in \mathcal{P}_{n}$ of the form

$$
p(x)=(x+b)^{n-m-k}(x-b)^{m} q(x), \quad q \in \mathcal{P}_{k},
$$

and for every

$$
\alpha \in\left[1,1+(2 n k)^{-1}\right]
$$


Lemma 15. Let $1 \leq k \leq n, 0 \leq m \leq 2 n-2 k$ be integers. We have

$$
|p(i \delta)| \leq \sqrt{c_{12}} \max _{t \in \mathbb{R}} \mid p(t \mid
$$

( $i$ denotes the imaginary unit) for every $p \in \mathcal{T}_{n}$ of the form

$$
p(t)=(\sin ((t+\gamma) / 2)))^{2 n-m-2 k}(\sin ((t+\gamma-\pi) / 2))^{m} q(t),
$$

with $q \in \mathcal{T}_{k}$ and $\gamma \in \mathbb{R}$, provided

$$
\delta \in\left(-(8 k n)^{-1 / 2},(8 k n)^{-1 / 2}\right) .
$$

Proof. The proof is quite similar to that of Lemma 7. Let

$$
\begin{aligned}
& \tilde{p}(t):=p(t) p(-t) \\
& \quad=4^{k-n}(\cos t-\cos \gamma)^{2 n-m-2 k}(\cos t+\cos \gamma)^{m} q(t) q(-t) .
\end{aligned}
$$

Since $q(t) q(-t) \in \mathcal{T}_{2 k}$ is an even trigonometric polynomimal, by the substitutions $x=\cos t$ and $b=\cos \gamma$, we obtain that

$$
\tilde{p}(t)=(x-b)^{2 n-m-2 k}(x+b)^{m} Q(x), \quad Q \in \mathcal{P}_{2 k},
$$

where $Q \in \mathcal{P}_{2 k}$. Let

$$
P(x):=(x-b)^{2 n-m-2 k}(x+b)^{m} Q(x) .
$$

Now (2.57) - (2.60) and Corollary 14 yield

$$
\begin{aligned}
& |p(i \delta)|^{2}=|p(i \delta) p(-i \delta)|=|\tilde{p}(i \delta)| \\
& =\left|(\cosh \delta+b)^{2 n-m-2 k}(\cosh \delta-b)^{m} Q(\cosh \delta)\right| \\
& \leq \max _{-1 \leq x \leq 1+\delta^{2}}|P(x)| \leq c_{12} \max _{t \in \mathbb{R}}|\tilde{p}(t)| \\
& =c_{12} \max _{t \in \mathbb{R}}|p(t) p(-t)| \leq c_{12} \max _{t \in \mathbb{R}}|p(t)|^{2},
\end{aligned}
$$

and the lemma is proved. 
Corollary 16. Let $1 \leq k \leq n, 0 \leq m \leq n, m+2 k \leq 2 n$ be integers. We have

$$
|p(z)| \leq \sqrt{c_{12}} \max _{t \in \mathbb{R}}|p(t)|
$$

for every $p \in \mathcal{T}_{n}$ of the form

$$
p(t)=(\sin (t / 2))^{2 n-m-2 k}(\sin ((t-\pi) / 2))^{m} q(t),
$$

with $q \in \mathcal{T}_{k}$, provided

$$
|\operatorname{Im}(z)| \leq(8 k n)^{-1 / 2}
$$

Proof. Let $\tilde{p}(t):=p(t-\gamma), \gamma \in \mathbb{R}$, and apply Lemma 15 .

Corollary 17. Let $1 \leq k \leq n, 0 \leq m \leq 2 n-2 k$ be integers. There is an absolute constant $c_{13}>0$ such that

$$
\max _{t \in \mathbb{R}}\left|p^{\prime}(t)\right| \leq c_{13} \sqrt{n k} \max _{t \in \mathbb{R}}|p(t)|
$$

for every $p \in \mathcal{T}_{n}$ of the form

$$
p(t)=\left(\sin \frac{t+\pi / 2}{2}\right)^{2 n-m-2 k}\left(\sin \frac{t-\pi / 2}{2}\right)^{m} q(t), \quad q \in \mathcal{T}_{k} .
$$

By the substitution $x=\sin t$, from Corollary 17 we deduce

Corollary 18. Let $1 \leq k \leq n, 0 \leq m \leq n-k$ be integers. We have

$$
\left|p^{\prime}(y)\right| \leq c_{13} \frac{\sqrt{n k}}{\sqrt{1-y^{2}}} \max _{-1 \leq x \leq 1}|p(x)|, \quad-1<y<1
$$

for every $p \in \mathcal{P}_{n}$ of the form

$$
p(x)=(x+1)^{n-m-k}(x-1)^{m} q(x), \quad q \in \mathcal{P}_{k},
$$

where $c_{13}>0$ is the same as in Corollary 17.

Now we are ready to prove the Theorem stated in Section 1.

Proof of the Theorem. The case $k=0$ follows from the case $k=1$, hence, without loss of generality, we may assume that $1 \leq k$. A simple compactness argument shows that for every $-1<y<1$ there is a $\tilde{p} \in \mathcal{P}(n, k)$ such that

$$
\frac{\left|\tilde{p}^{\prime}(y)\right|}{\max |\tilde{p}(x)|}=\sup _{n \in \mathcal{D}(n k)} \frac{\left|p^{\prime}(y)\right|}{\max |p(x)|}
$$


Observe that $\tilde{p}$ cannot attain its maximum modulus on $[-1,1]$ at $y$, otherwise $\tilde{p}^{\prime}(y)=0$, a contradiction. We will show that $\tilde{p}$ has at most $k+1$ zeros (by counting multiplicities) different from \pm 1 . First we prove that $\tilde{p}$ has only real zeros. Indeed, if $\tilde{p}(a)=0$ for an $a \in \mathbb{C} \backslash \mathbb{R}$, then $\tilde{p}(\bar{a})=0$, and a simple calculation shows that, with a sufficiently small $\epsilon>0$,

$$
p_{\varepsilon}(x):=\tilde{p}(x)\left(1-\varepsilon \frac{(x-y)^{2}}{(x-a)(x-\bar{a})}\right)
$$

is in $\mathcal{P}(n, k)$ and contradicts the extremality of $\tilde{p} \in \mathcal{P}(n, k)$.

Next we show that $\tilde{p}$ can not have more than one zero (by counting multiplicities) in $\mathbb{R} \backslash[-1,1]$. Indeed, if there are $a \neq b \in \mathbb{R} \backslash[-1,1]$ such that $\tilde{p}(a)=\tilde{p}(b)=0$, or there are $a=b \in \mathbb{R} \backslash[-1,1]$ such that $\tilde{p}(a)=\tilde{p}^{\prime}(a)=0$, then the polynomial

$$
p_{\varepsilon}(x):=\tilde{p}(x)\left(1-\varepsilon \operatorname{sign}(a b) \frac{(x-y)^{2}}{(x-a)(x-b)}\right)
$$

with sufficiently small $\varepsilon>0$ is in $\mathcal{P}(n, k)$, and it contradicts the extremal property of $\tilde{p}$. Since $\tilde{p} \in \mathcal{P}(n, k), \tilde{p}$ has at most $k+1$ zeros (by counting multiplicities) different from \pm 1 , hence Corollary 18 and the extremal property of $\tilde{p} \in \mathcal{P}(n, k)$ yield

$$
\left|p^{\prime}(y)\right| \leq c_{13} \frac{\sqrt{n(k+1)}}{\sqrt{1-y^{2}}} \max _{-1 \leq x \leq 1}|p(x)|
$$

for every $p \in \mathcal{P}(n, k)$. The uniform bound

$$
\max _{-1 \leq x \leq 1}\left|p^{\prime}(x)\right| \leq 9 n(k+1) \max _{-1 \leq x \leq 1}|p(x)|
$$

for every $p \in \mathcal{P}(n, k)$ is proved in [ER2, Corollary 1.3] extending [BO]. Other proofs of (2.76) are given in [ER3, Theorem 1] and [BO-ER, Theorem 3.4] with slightly larger multiplicative constants than 9. By (2.75) and (2.76) the theorem is proved. 


\section{REFERENCES}

[BO] P. Borwein, Markov's inequality for polynomials with real zeros, Proc. Amer. Math. Soc. 93 (1985), 43-47.

[BO-ER] P. Borwein and T. Erdélyi, Remez, Nikolskii and Markov type inequalities for generalized non-negative polynomials with restricted zeros, Constr. Approx. (to appear).

[ER1] T. Erdélyi, Markov and Bernstein type inequalities for certain classes of constrained trigonometric polynomials on an interval shorter than the period, Studia Sci. Math. Hung., 25 (2) (1990), 3-25.

[ER2] T. Erdélyi, Pointwise estimates for derivatives of polynomials with restricted zeros, Coll. Math. Soc. J. Bolyai, 49, A. Haar Mem. Conf., Budapest, Hungary, 1985, North Holland, Amsterdam, 1986, pp. 329343.

[ER3] T. Erdélyi, Bersntein-type inequality for the derivative of constrained polynomials, Proc. Amer. Math. Soc., 112 (3) (1991), 829-838.

[ER4] T. Erdélyi, Markov-type estimates for certain classes of constrained polynomials, Constr. Approx. 5 (1989), 347-356.

[ER-SZA] T. Erdélyi and J. Szabados, Bernstein type inequalities for a class of polynomials, Acta Math. Hung. 53 (1-2) (1989), 237-251.

[ERDÖS] P. Erdős, On extremal properties of the derivatives of polynomials, Ann. of Math. 41 (2) (1940), 310-313.

[LO1] G. G. Lorentz, "Approximation of Functions," Hold, Rinehart and Winston, New York, 1966.

[LO2] G. G. Lorentz, The degree of approximation by polynomials with positive coefficients, Math. Ann. 151 (1963), 239-251.

[MA] A. Máté, Inequalities for derivatives of polynomials with restricted zeros, Proc. Amer. Math. Soc. 88 (1981), 221-225.

[SCH] J. T. Scheick, Inequalities for derivatives of polynomials of special type, 
[SZA] J. Szabados, Bernstein and Markov type estimates for the derivatives of a polynomial with real zeros, in "Functional Analysis and Approximation," P. L. Butzer, B. Sz.-Nagy and E. Görlick, eds., Birkhäuser, Basel, 1981, pp. 177-188.

[SZA-VA] J. Szabados and A. K. Varma, Inequalities for derivatives of polynomials having real zeros, in "Approximation Theory III," E. W. Chaney, ed., Academic Press, New York, 1980, pp. 881-888. 